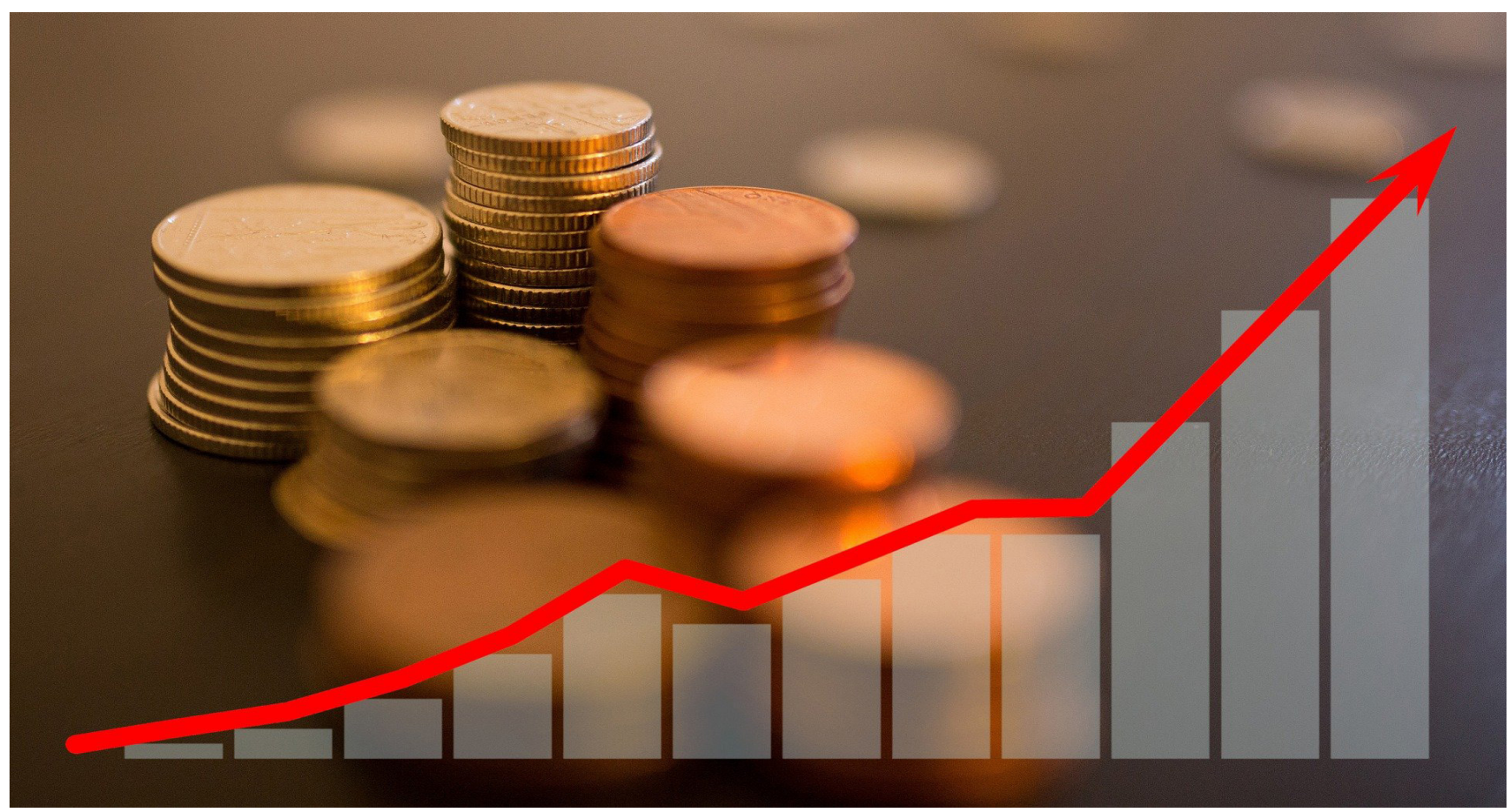

\title{
Beneficios tributarios $y$ financieros para empresas vinculadas a zonas francas en Cúcuta
}

\section{Tributary and financial benefits for companies linked to free zones in Cúcuta}

\section{Blanca Liliana Carrascal-Velásquez}

Facultad de ciencias Empresariales, grupos de investigación GTN y GIPAB, Correo Electrónico: bl_velasquez@fesc.edu.co, Fundación de estudios superiores Comfanorte, Ocaña-Colombia,

Maria Fernanda García

Estudiante de Negocios internacionales, est_mf_garcia@fesc.edu.co, Fundación de Estudios Superiores Comfanorte, Cúcuta, Colombia.

Tibizay Castellanos-Diaz

Estudiante de Negocios Internacionales, est_t_castellanos@fesc. edu.co Fundación de Estudios Superiores Comfanorte, Cúcuta, Colombia.
Johann Fernando Hoyos-Patiño

Facultad de ciencias agrarias y del ambiente/Departamento pecuario, GI@S y GIPAB, jfhoyosp@ufpso.edu.co Universidad Francisco de Paula Santander, Ocaña-Colombia,

Lilian Natalia Sayado-Velasquez

Estudiante administracion financiera, velasqueznati29@gmail.com, Universidad de Santander, Cúcuta, Colombia 


\section{Resumen}

Las zonas francas desde sus inicios han sido una herramienta empleada para la captación de inversión, generación de empleo y competitividad en las regiones donde se encuentra, entre otros factores que ayuden a la economía colombiana. Por lo cual, el objetivo del presente escrito es presentar los beneficios que trae para las empresas el estar establecidas en una Zona Franca y el campo que esta les abre a nuevos horizontes que buscan ser capturados. La metodología que se utilizó para llevar a cabo la finalidad de la investigación fue cualitativa ya que el medio que se utilizó para recolección de información fue una entrevista hecha al director de operaciones de la Zona Franca de Cúcuta (Hector Zapata Franco), con lo cual se buscaba dar respuesta a los objetivos planteados al comienzo de la averiguación. A principios del año en curso, estas se vieron afectadas por un fenómeno que no estaba en panorama de nadie pero que llego para cambiarlo todo, por lo cual se tuvieron que tomar decisiones que en varios aspectos afecto a muchas de las empresas que estaban consolidadas en estas, por lo que se busca mencionar que fue lo que sucedió con la tan llegada pandemia a la nación y los ajustes que trajo consigo.

Palabras clave: Beneficios tributarios, competitividad, empleo, empresa, inversión, pandemia, Zona Franca.

\section{Abstract}

Free zones since their inception have been a tool used for investment capture, job creation and competitiveness in the regions where it is located, among other factors that help the Colombian economy. Therefore, the objective of this writing is to present the benefits that it brings for companies to be established in a Free Zone and the field that it opens them to new horizons that seek to be captured. The methodology used to carry out the purpose of the investigation was qualitative since the medium used for information collection was an interview with the director of operations of the Free Zone of Cúcuta (Hector Zapata Franco), which sought to respond to the objectives set at the beginning of the investigation. At the beginning of the current year, these were affected by a phenomenon that was not in anyone's picture but that came to change everything, so decisions had to be made that in various respects affected many of the companies that were consolidated in them, so it is sought to mention that it was what happened with the much-coming pandemic to the nation and the adjustments it brought with it.

Keywords: Tax benefits, competitiveness, employment, business, investment, pandemic, Free Zone. 


\section{6}

\section{Introducción}

Los hechos han demostrado que las zonas francas (ZF) son un medio importante para mejorar la competitividad nacional, y se han convertido en un captador para atraer inversiones, generar empleo, fomentar la diversificación, y la transferencia de tecnología principalmente para impulsar el desarrollo de aquellas empresas que buscan disminuir sus costos, generar utilidades y confrontar desafíos en un mundo tan cambiante y sobretodo en un camino tan dinámico, competitivo como lo es el comercio alrededor del mundo de acuerdo con Mora et al. (2019).

Una ZF se refiere a un área geográfica definida dentro del territorio de Colombia, de acuerdo con regulaciones especiales relacionadas con impuestos, aduanas y comercio exterior, el área ha desarrollado actividades industriales o comerciales de bienes y servicios Rivera et al., (2019). ProColombia señaló que el régimen franco cuenta con ventajas competitivas como las facilidades para importar y exportar con exención de IVA y aranceles. También se cuenta con exención de IVA para materias primas, insumos y bienes terminados adquiridos en el territorio aduanero nacional y tienen todo el potencial en el aprovechamiento de los 16 acuerdos comerciales con acceso a un mercado de más de 1.600 millones de consumidores.

A causa de esto hacen un gran aporte en la región donde se encuentran establecidas ya que son un factor clave para el incremento de importaciones y exportaciones, oportunidades de empleo, lo que genera una percepción positiva de lo que esta pueda ofrecer a esa empresa que busque todas estas metas, Carrascal et al., (2020). El objetivo primordial de esta investigación es presentar los beneficios que trae para las empresas el estar establecidas en la ZF, como analizar el impacto que ha tenido para la economía del país en su propósito de generador de inversión y empleo, también cabe mencionar los contratiempos que le presento la actual pandemia.

A finales de la década de los 50 por la importancia de juntar la generación de empleo y el desarrollo de las exportaciones manufactureras, se introdujo en el mundo el concepto de ZF. Buscando un nuevo concepto, la primera ZF fue establecida en Irlanda en 1959 y fue tomada como modelo por diferentes países alrededor del mundo. Herrera et al., (2018).

Y en vista de lo anterior durante los últimos años las ZF han sido un instrumento muy útil para incentivar el comercio exterior de los países, a causa de que cuenta beneficios en lo que respecta a materia aduanera y tributaria que les permiten a las empresas tener un mejor funcionamiento y en otra instancia la disminución de algunos costos e impuestos, Chaustre et al., (2018). Con respecto a lo ya mencionado se dio inicio en Colombia la implementación de las ZF en 1958 con la apertura de la primera de estas ubicada en barranquilla, gracias a ella años después se darían a conocer 5 más las cuales fueron: Santa Marta, Palmaseca, Cúcuta, Buenaventura y Cartagena.

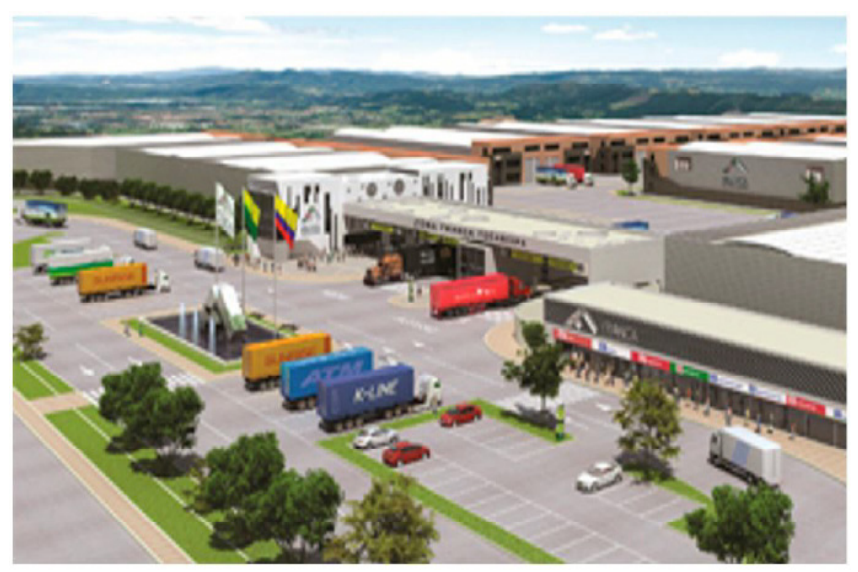

Imagen 1, obtenida de: https://www.legiscomex.com/Documentos/articulozonas-francas-2015-rci301

Por lo tanto, en la ley 1004 del 2005 se define a la ZF como: el área geográfica delimitada 
dentro del territorio nacional, donde se desarrollan actividades industriales de bienes y de servicios, bajo una normatividad especial en materia tributaria, aduanera y de comercio exterior. Las mercancías ingresen a esta área se consideran fuera del TAN para la modalidad de impuestos a importaciones y exportaciones. Sistema Único de información normativo (2005). Esta ley presentada por el gobierno, tiene como finalidad el marco normativo de las ZF y la condición para gozar de este, es que la empresa obtenga compromisos de inversión y generación de empleo.

En consecuencia, la creación de esta figura ha traído consigo la realización de un objetivo que lleve a la generación de empleo. Según el más reciente informe estadístico de la ANDI para el primer trimestre del año 2019 se demostró que se generaron 165.538 empleos, de los cuales 56.676 son directos, 80.806 son indirectos y 28.056 son relacionados. Vita (2020), lo que significa que se está trabajando en pro del mejoramiento de la economía del país y del nivel de vida de ciudadanos que antiguamente se encontraba con uno de los principales problemas de nuestra sociedad que es la falta de empleo.

Sánchez \& Segura (2020), en la actualidad, a principios de año las ZF se vieron afectadas a causa de la llegada del covid-19 a Colombia causando un fuerte impacto en los temas de comercio exterior ya que se tuvieron que detener por un tiempo estas actividades. La Pandemia COVID-19 han afectado la actividad del sector productivo colombiano y así mismo, las actividades de comercio internacional, perturbando de manera negativa la importación y exportación de bienes, por lo cual se buco implementar medidas por parte del gobierno colombiano. Por otra parte el Ministerio de comercio, industria y turismo (2020). Afectando este factor a las empresas ubicadas en las ZF debido a que muchas de ellas exportan en gran masa y con esto perturbando al país en la caída de los precios de los productos básicos.
La importancia de esta temática recae sobre el incentivo para generar empleo, ya que desde siempre en las empresas prima el minimizar sus costos y aumentar sus utilidades, Álvarez (2015). buscando así se competitivos y sobrevivir en un mundo tan cambiante como en el que vivimos hoy en día. Dando lugar a la ZF que es una opción factible para que las empresas puedan cumplir sus objetivos. El investigar esta figura recae en que las ZF representan oportunidades para las empresas que quieran fortalecer su actividad en el país, contribuyendo con inversión, innovación y generación de empleo. Lo que significaba un divisor estable para aquellos inversionistas permitiéndoles tener una perspectiva favorable a larga instancia.

El régimen franco es un pilar importante de la competitividad nacional, porque permite atraer inversión extranjera directa (IED), para tener el impacto que se busca con los privilegios en conceptos de inversión y generación de empleo. De acuerdo con Ramos \& Rodríguez (2011). esto siendo un beneficio muy importante ya que traería al país tecnología adecuada para los procesos de producción, que haya más exportaciones y permitiendo alcanzar un crecimiento económico que como nación, es el principal objetivo.

De acuerdo con Pinzón \& Lora (2015) Las ZF se han consolidado como dinamizadores claves para impulsar la economía del país; según la ANDI, en los últimos años se ha percibido una inversión de 39 billones de pesos, asimismo, las exportaciones anualmente que se recibieron sumaron un valor total de 2 billones de dólares. Por ejemplo, en "abril de 2019 las exportaciones de mercancías desde las zonas francas colombianas mostraron un aumento de $35,4 \%$, al pasar de US $\$ 253,6$ millones FOB en abril 2018 a US\$343,2 millones FOB en el mismo mes de 2019". Por otra parte la Zona Franca Bogotá para el 2019 en el último reporte de las ZF se puso evidenciar que las esas zonas son realmente generadores de 


\section{8}

inversionistas como lo evidencia la anterior cita mostrándonos que cada año aumenta y genera mucho más movimiento comercial (exportaciones), lo que implica no sólo un incremento de la competitividad del país, sino una oportunidad de desarrollo para la sociedad en donde la zona se encuentra localizada, Arévalo \& Arévalo (2019)

De los grandes retos de la ZF es el incentivar a las pequeñas, medianas y grandes empresas que pongan su voto de confianza en ellas para que puedan anhelar a grandes mercados para que puedan ser vistos y alcancen inversión extranjera y promuevan el desarrollo de la región. Otros beneficios que brinda las ZF son: seguridad, telecomunicaciones, centro de salud, guardería, cafetería y transporte para empleados, etc. (Legiscomex) viéndose así su responsabilidad y preocupación por sus empleados que son un factor esencial para la realización de su objeto social.

En Colombia, el número de ZF ha aumentado como pilar del desarrollo regional, transferencia de tecnología, y generadoras de industrias y servicios para el mercado nacional e internacional, Alvarez \& Suarez (2016). En términos más generales, contribuyen al crecimiento económico y por ende se han convertido en creadoras de ingresos para el estado al aportar recursos a través de los impuestos directos (como el de renta), e indirectos (IVA y arancel de aduanas). Así mimos indican Álvarez \& Lora (2015) también se suman los acuerdos de libre comercio que abren las puertas a nuevos mercados y que permite hacer presencia en otros países para así seguir ayudando al país en pro de un crecimiento económico que pueda servir de apoyo para conseguir una nación mejor donde los ciudadanos puedan lograr un estilo de vida bueno que les permita estar al alcance de una buena educación, salud, entre otros factores.

\section{Materiales y métodos}

Para Bernal, (2010). El método cualitativo hace alusión a indagar acerca de casos concretos de fenómenos sociales, con la finalidad de cualificar y describir este a partir de ciertos rasgos determinados. Busca entender una situación con la finalidad de darle solución o tratar de razonar esta.

El enfoque a utilizar en este proyecto se verá reflejado en un enfoque cualitativo puesto que por medio de una entrevista se buscará analizar el pensamiento y comportamiento de una persona especialista en la temática para llegar a la finalidad de la investigación, además que también se regirá un alcance descriptivo con el objetivo de examinar el fenómeno de la investigación.

\section{Resultados y análisis}

A continuación, se mostrará cuáles fueron las preguntas y las respuestas dadas al conocedor de la temática tratada en la presente investigación, el cual fue Hector Zapata Franco, actual director de operaciones de la Zona Franca de Cúcuta quien fue un factor muy importante para poder resolver la finalidad de la averiguación.

1. ¿Por qué una empresa debería establecerse en una ZF?

Esto depende de las características de la empresa y del ejercicio que pretenda realizar; si su actividad es industrial y elaboran productos, estas empresas tienen mayor beneficio, enfocado principalmente en la disminución de la contribución del tributo de renta que es lo que más afecta los rendimientos de las inversiones de los empresarios, entonces las ZF tienen incentivo de una disminución de renta al 20\%, fuera de esta la renta de estos empresarios es de un 33\%. Si bien las zonas francas brindan oportunidades y es un espacio 
propicio para el desarrollo empresarial, en especial de las pequeñas y medianas empresas. 2. ¿Cuál ha sido el impacto que ha tenido la pandemia en la ZF de Cúcuta y qué medidas se han tomado para sobrellevar este fenómeno? Si bien la pandemia afecto la ZF de Cúcuta en la disminución de operaciones para algunos usuarios, también otros se vieron impactados de forma positiva porque sus productos están dentro de las industrias que están exentas de las medidas de las restricciones y otros usuarios tenían productos que servían o de una u otra forma se podían emplear con fines de la prevención del covid, entonces esto sencillamente se tradujo en beneficios para ellos, pero realmente el impacto negativo sobre la ZF viene es desde el cierre de frontera para carga que es donde el efecto negativo se ha podido evidenciar en la ZF, el de la pandemia no fue tan grave y alto porque ya venía con un problema derivado del cierre Colombia-Venezuela y Venezuela-Colombia .

3. ¿Cuál ha sido el impacto que han tenido la ZF para la economía colombiana en los últimos años?

Su impacto ha sido positivo para el país, ya que cumplen sus objetivos principales que son la generación de empleo e inversión y se puede evidenciar en las cifras de las inversiones que se han tenido en las ZF que son aproximadamente 47 millones de dólares que se tenían invertido en estas finalizando el 2017, entonces son muy buenas cifras para el país y ha generado muchísimos empleos y pues ha sido un impacto positivo no solo para Colombia en general sino para los lugares donde estas se establecen. La balanza de las ZF en cuanto a importación-exportación es positiva.

4. ¿Qué huella ha tenido la ZF de Cúcuta con el propósito de la generación de empleo y captación de inversión?
Si bien la ZF de Cúcuta ha tenido inconvenientes derivados de los problemas fronterizos y además porque esta se acostumbró a ser muy comercial, solo aduano de paso, las mercancías llegaban y no se les hacía ningún proceso productivo a estas y continuaban, ahora se presenta un escenario distinto, el cierre de frontera fue una oportunidad buena para que los productores locales ya que no tienen que competir con los productos venezolanos y eso ha permitido tener un incremento en la producción en los usuarios industriales que son los que generan empleo y comienzan a generar estas economías de escala. Actualmente la ZF de Cúcuta aun con todos los inconvenientes que se vienen presentado esta alrededor de los 300 empleos que es una buena cifra para una ZF que tiene poco movimiento y que realmente es una de las más pequeñas del país, entonces el impacto que ha tenido para Cúcuta y la región ha sido positivo.

5. Usted como director de operaciones de la ZF de Cúcuta, ¿qué estrategias propondría para que empresas en la ciudad se animaran a formar parte de esta?

Aquí hay que entender que la ZF es una plataforma que permite el desarrollo de las regiones donde ella se establezca, mi proposición es que las empresas se instalaran en la ZF por el tema de la formalidad que es un factor que afecta bastante a la región de Norte de Santander y la economía del país que es la creación de empleos sin lo mínimo de la seguridad social y que se bajen los impuestos en muchas de estas empresas informales, esto de una forma u otra forma termina perjudicando a las empresas que están haciendo las cosas bien, entonces propondría seguir apoyando y acompañando a las empresas que tienen ese potencial para estar en la ZF y hacerles el acompañamiento para que tengan un buen desarrollo del régimen franco. 


\section{0}

6. En la actualidad, ¿qué funciones están cumpliendo las Zonas Francas?

Las zonas francas están haciendo y jugando un rol muy importante en el país, hay empresas que son realmente productivas y eficientes en el manejo de los recursos y cada vez los estándares son más altos para las empresas que hacen parte de régimen franco, permitiendo que hoy hayan empresas certificadas, que tienen buenas practicas, un uso eficientes de los recursos, altamente productivas y que están representando no solo en el escenario naciones sino en escenarios internacionales los productos del país, entonces han sido un bueno ejercicio. En la actualidad las zonas francas vienen cumpliendo, realizando muy buenas cosas por el desarrollo empresarial del país, así que sus funciones y roles han estado bien vistas, si bien la visión inicial de las zonas francas está dirigida a las exportaciones a estado en el ámbito nacional muy fuerte, posicionadas.

7. ¿Cuáles son las expectativas que tiene la ZF luego de que pase esta epidemia?

Lo primero es retomar ya que se espera que la pandemia termine pronto, igual las responsabilidades, compromisos y obligaciones de las zonas francas continúan. Ahora se viene un reto para la ZF de Cúcuta y para muchas del país que es el obtener la autorización de prórroga, lo que significa que a estas zonas francas les permitan continuar por un periodo mayor (aproximadamente 30 años), entonces para lograr eso hay que lograr unas certificaciones, presentar un plan de desarrollo de las zonas francas. Eso es lo que se aproxima para la ZF y es un proceso bueno porque lo que hace es fortalecerlas más.

Gracias a esta entrevista que nos brindó el director de operaciones de las zona franca de Cúcuta se pudieron aclarar dudas, proveer mayor información acerca de que ha estado representando las zonas francas para el país en los últimos años y sobretodo que es lo que se viene para esta una vez acabe esta pandemia que se vive que a fin de cuentas es sumamente bueno e importante porque estas pueden seguir desarrollando sus actividades y brindar beneficios locales, regionales y nacionales que ayuden en pro de un crecimiento económico que favorece a toda la sociedad.

\section{Conclusiones}

Las ZF buscan constantemente la captación de inversión que es un factor que impacta positivamente por una parte a las empresas a causa de que genera empleos y busca impulsar el desarrollo e innovación de las actividades que están realicen haciendo que abarquen mayores mercados y por otra parte otorga financiamiento al país, contribuyendo en su desarrollo económico. Esta herramienta permite desarrollar la economía local, regional y nacional por medio de la creación de empleos que reconocen mejorar la calidad de vida de los ciudadanos que en esta habitan.

Por lo que la principal finalidad de esta investigación era el dar a conocer este importante instrumento para aquellas personas empresarias y próximas a serlo, para que visualizaran la importancia que reside el establecerse en una ZF, los beneficios que genera y las oportunidades que presenta como el incursionar en mercados internacionales permitiéndoles así el ser reconocidos en otros caminos. Gracias a los resultados que se recopilaron del director de operaciones de la ZF de Cúcuta se pudo visualizar el propósito por el cual fue creada la presente investigación, se pudieron dar respuesta a los objetivos planteados en un inicio, se proveyó información necesaria para poder entender la razón de ser de una herramienta de ayuda para aquellos empresarios y emprendedores que buscan salir a flote en un mercado donde continuamente brotan marcas insurgentes. 


\section{Referencias}

Alvarez, E. P., \& Suarez, J. E. L. S. L. (2016). La generación de beneficios sociales y económicos de las zonas francas en Colombia. FACE: Revista de la Facultad de Ciencias Económicas y Empresariales, 15(2), 53-67. Obtenido de: http:// revistas.unipamplona.edu.co/ojs_viceinves/ index.php/FACE/article/view/1907

Álvarez-Díaz, R. (2015). Zonas francas como generadoras de empleo en Colombia. Obtenido de: https://pdfs.semanticscholar.org/5eb9/ cca47e38497d7126881b7640f89e10d2d4a7.pdf

Alvarez Pinson, E., \& Lora Suarez, J. E. (2015). La generación de beneficios sociales y económicos de las Zonas Francas en Colombia. Revista de la facultad de ciencias económicas y empresariales, 14 Recuperado de: https://n9.cl/46ar2

Arévalo Luna, G. A., \& Arévalo-izarazo, G. A. (2019). Las zonas francasen Colombia: desarrollo empresarial y regional, 2009-2016. Apuntes del CENES, 38(68), 151-184. Obtenido de: http:// www.scielo.org.co/scielo.php? pid $=\mathrm{S} 0120$ $30532019000200151 \& \mathrm{script}=\mathrm{sci}$ abstract\&tlng=pt

Bernal, C. (2010). En C. A. Torres, Metodología de la investigación (pág. 320). Bogotá: Pearson. Recuperado de: https://n9.cl/fvc6.

Carrascal, B. L. V., Patiño, J. F. H., Villamizar, D. A. H., Velasquez, L. N. S., Velásquez, J. E. S., \& Yuncosa, J. A. V. (2020). (DIE)MODELO PARA EL DISEÑO DE IDEAS DE EMPRENDIMIENTO. FAGROPEC-Facultad de Ciencias Agropecuarias, 12(1), 52-64. Obtenido de: https://www.uniamazonia.edu.co/ revistas/index.php/fagropec/article/view/1816

Chaustre, J. B., Cárdenas, A. G., Laureano, V. I., \& Carrascal, B. L. V. (2018). Análisis de aceptación procesos de textiles eco amigables en la industria de la moda en la ciudad de
Cúcuta. Revista CONVICCIONES, 5(9), 4852. Obtenido de: https://www.fesc.edu.co/ Revistas/OJS/index.php/convicciones/article/ view/265

Herrera Bernal, A. R., Pérez Gonzales, I. A., \& Garavito Álvarez, K. M. (26 de Noviembre de 2018). Uniagustiniana. Obtenido de Uniagustiniana: Recuperado de: https:// n9.cl/7jqgs

Legiscomex. (s.f.). Obtenido de Legiscomex: Recuperado de: https://n9.cl/h71o

Mora-Villamizar, D. A., Morales-Pérez, K. T., Barrientos-Monsalve, E. J., \& VelázquezCarrascal, B. L. (2019). Análisis de la competitividad entre las empresas los olivos y la esperanza en Cúcuta, Norte de Santander-según las cinco fuerzas de Michael Porter. Revista CONVICCIONES, 6(11), 6975. Obtenido de: https://www.fesc.edu.co/ Revistas/OJS/index.php/convicciones/article/ view/418

Ministerio de comercio, industria y turismo. (2020). Obtenido de Ministerio de comercio, industria y turismo: Recuperado de: https:// n9.cl/dxebn

Sistema Único de Informacion normativa. (31 de Diciembre de 2005). Obtenido de Sistema Único de Informacion normativa: Recuperado de: https://n9.cl/rx9sa

Ramos, J., \& Rodríguez, K. (2011). Zonas francas en Colombia: beneficios tributarios en el impuesto de renta. Banco de la República. Obtenido de: https://www.banrep.gov.co/ docum/ftp/borra657.pdf

Rivera-Lema, A. F., Urbina-Ramírez, N. G., Silva-Pérez, I. E., Barrientos-Monsalve, E. J., \& Velázquez-Carrascal, B. L. (2019). Analysis of the economic revenue management of the graphic designer in Colombia. Revista 
32

CONVICCIONES, 6(11), 23-29. Obtenido de: https://www.fesc.edu.co/Revistas/OJS/index. $\mathrm{php} /$ convicciones/article/view/383

Sánchez Caro, Y., \& Segura Villamil, Y. (2020). Economía colombiana en tiempos de Covid-19. Obtenido de: https://repository.ucatolica.edu.co/ handle/10983/24657

Pinzón Alvarez, E., \& Lora Suarez, J. E. (2015). La generación de beneficios sociales y económicos de las zonas francas en Colombia. Obtenido de: https://expeditiorepositorio.utadeo.edu.co/ handle/20.500.12010/8985

Vita Meza, L. (20 de Marzo de 2020). Las Zonas Francas generan más de 165.500 empleos, casi el doble que hace 10 años, pág. Recuperado de: https://n9.cl/bq2xy. 